\section{( OPEN ACCESS}

RESEARCH PAPER

\title{
Bone health in Parkinson's disease: a systematic review and meta-analysis
}

\author{
Kelli M Torsney, ${ }^{1}$ Alastair J Noyce, ${ }^{2}$ Karen M Doherty, ${ }^{2}$ Jonathan P Bestwick, ${ }^{3}$
Ruth Dobson ${ }^{4}$ Andrew J Lees ${ }^{2}$
}

- Additional material is published online only. To view please visit the journal online (http://dx.doi.org/10.1136/ jnnp-2013-307307).

${ }^{1}$ Emergency Department, West Middlesex Hospital, London, UK

${ }^{2}$ Reta Lila Weston Institute of Neurological Studies, UCL Institute of Neurology, London, UK

${ }^{3}$ Wolfson Institute of Preventive Medicine, Queen Mary University of London, London, UK

${ }^{4}$ Blizard Institute, Centre for Neuroscience and Trauma, Queen Mary University of London, London, UK

Correspondence to Professor Andrew J Lees, Reta Lila Weston Institute of Neurological Studies, UCL Institute of Neurology, 1 Wakefield Street, London WC1N 1PJ, UK; andrew.lees@ ucl.ac.uk

Received 23 November 2013 Revised 14 January 2014 Accepted 28 January 2014 Published Online First 11 March 2014

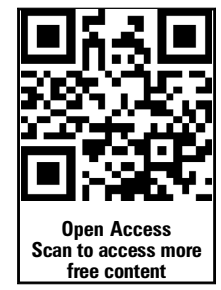

CrossMark

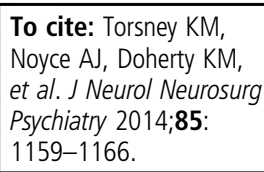

\section{ABSTRACT}

Objective Parkinson's disease (PD) and osteoporosis are chronic diseases associated with increasing age. Single studies have reported associations between them and the major consequence, namely, increased risk of fractures. The aim of this systematic review and metaanalysis was to evaluate the relationship of PD with osteoporosis, bone mineral density (BMD) and fracture risk.

Methods A literature search was undertaken on 4 September 2012 using multiple indexing databases and relevant search terms. Articles were screened for suitability and data extracted where studies met inclusion criteria and were of sufficient quality. Data were combined using standard meta-analysis methods.

Results 23 studies were used in the final analysis. PD patients were at higher risk of osteoporosis (OR 2.61; $95 \% \mathrm{Cl} 1.69$ to 4.03 ) compared with healthy controls. Male patients had a lower risk for osteoporosis and osteopenia than female patients (OR $0.45 ; 95 \% \mathrm{Cl} 0.29$ to 0.68$)$. PD patients had lower hip, lumbar spine and femoral neck BMD levels compared with healthy controls; mean difference, $-0.08,95 \% \mathrm{Cl}-0.13$ to -0.02 for femoral neck; $-0.09,95 \% \mathrm{Cl}-0.15$ to -0.03 for lumbar spine; and $-0.05,95 \% \mathrm{Cl}-0.07$ to -0.03 for total hip. PD patients were also at increased risk of fractures (OR 2.28; $95 \% \mathrm{Cl} 1.83$ to 2.83 ).

Conclusions This systematic review and meta-analysis demonstrate that PD patients are at higher risk for both osteoporosis and osteopenia compared with healthy controls, and that female patients are at greater risk than male patients. Patients with PD also have lower BMD and are at increased risk of fractures.

\section{INTRODUCTION}

Parkinson's disease (PD) is a neurodegenerative disorder which affects $1 \%-2 \%$ of the UK population over 65 years. ${ }^{1}$ Osteoporosis describes a reduction of bone mineral density (BMD) which places those affected at increased risk of fragility fractures particularly those involving the hip, wrist and spine. ${ }^{2}$ Multiple factors contribute to the development of osteoporosis including age, gender, height, weight, family history, smoking status and vitamin D levels. ${ }^{3}$ Poor bone health results in significant morbidity and mortality, as well as being detrimental to quality of life. ${ }^{2}$ There is increasing evidence to suggest neurological conditions including epilepsy, multiple sclerosis, dementia and PD are associated with an excess rate of osteoporosis and fracture risk. ${ }^{45}$
The Global Longitudinal Study of Osteoporosis in Women (GLOW) study found PD to be the strongest single contributor to fracture risk compared with other studied factors. ${ }^{4}$ Gait impairment, postural instability and falls, polypharmacy and reduced BMD all contribute to fracture risk in PD. ${ }^{6}$ Vitamin D deficiency with secondary hyperparathyroidism may contribute to low BMD but disease duration and severity, age and low body mass index are also implicated.

BMD is traditionally measured using dual $\mathrm{x}$-ray absorbance spectrometry (DEXA), which measures bone density per unit area. The results are normalised to age- and gender-matched members of the general population, generating a Z-score. Normalisation against a population of young healthy adults gives a T-score. Osteoporosis is defined as a T-score $<-2.5 \mathrm{SDs}$ from the norm and osteopenia as a T-score between -1 and -2.5 SDs. $^{2}$

The aim of this systematic review and meta-analysis was to summarise and combine the published data on the association of PD with fracture risk and BMD.

\section{METHODS}

\section{Search strategy}

The PRISMA 2009 guidelines for systematic review and meta-analysis were followed throughout the study. ${ }^{8}$ A strategy was developed to search PubMed, SciVerse Scopus and Google Scholar with the following terms: 'Osteoporosis and PD', 'Osteopenia and PD', 'Fracture and PD' and 'Bone health and PD'. The search was carried out on 4 September 2012.

The search was restricted to English articles. Article titles and abstracts were reviewed for relevance pertaining to the following three items of interest: (1) risk of osteoporosis/osteopenia in PD; (2) BMD in PD; and (3) fracture risk in PD. Articles were excluded on the basis of title or abstract if they were not relevant to either bone health or fracture risk in PD. The full articles of relevant studies were obtained and reviewed. Reference lists of relevant articles were hand searched for any additional references not picked up by the electronic database searches, as were the reference lists of existing meta-analyses and any review articles identified through the original database search. Three reviewers (KMT, AJN and KMD) reviewed and filtered the articles at each stage and differences in opinion were resolved through discussion. 


\section{Inclusion criteria}

Published studies were included if the following criteria were fulfilled: (1) observational studies with either a cohort or casecontrol design; (2) cases were patients with PD according to standard clinical criteria, for example, Queen Square Brain Bank Criteria; ${ }^{9}$ (3) controls were healthy or had no history of neurological disease; (4) original data were reported; (5) BMD was measured using DEXA scans.

\section{Exclusion criteria}

Abstracts and published conference proceedings, editorials, commentaries, review articles, case reports, meta-analyses and letters that did not report new data were all excluded. We also excluded studies that: (1) reported on the management of fractures; (2) reported fractures prior to the onset of PD; (3) did not provide adequate details of the control group; (4) reported data for cases not fulfilling clinical criteria for PD; (5) reported data for factors other than BMD or fracture, for example, falls, body mass index, dietary intake or sunlight exposure; (6) were randomised controlled trials; (7) reported risk estimates other than relative risks (RR)/odds ratios (ORs)/hazard ratios (HRs), such as mortality rate and standardised hospitalisation ratios.

\section{Data handling}

Three authors (KMT, AJN and KMD) independently collected and tabulated the data into an electronic spread sheet, under the following headings: PubMed ID, author, year of publication, year of study, population studied, study design, number of males, number of females, mean ages and the results for the specific factor of interest (eg, number with fractures, number with osteoporosis, average BMD).

If case-control studies reported data for more than one control group, we used the control group most representative of the general population. In studies that had no calculated risk estimate (RR/OR/HR), we reviewed the crude data and calculated an OR where possible. In studies that reported both crude and adjusted OR, the adjusted figure was used. Where two studies with the same population and overlapping follow-up periods were found, the study with greatest number of subjects was used. If population sizes were equal, the most recent study was used. Where data were not clearly reported, the corresponding author of the article was contacted and data were made available. After application of the above methods, the quality of the remaining articles was assessed using the Newcastle Ottawa Scale (NOS). ${ }^{10}$ We set a predetermined threshold of study quality as a score of 7 out of 9 and excluded any studies that scored below this threshold.

\section{Statistical analysis}

Where a factor of interest was reported by two or more studies in a consistent manner, these were combined using standard meta-analysis methods to generate a pooled OR and $95 \%$ confidence intervals (CIs) for each factor. The OR was used as an estimate of $\mathrm{HR}$ or $\mathrm{RR}$ in relevant studies. Heterogeneity between studies was assessed using the $\mathrm{I}^{2}$ statistic and, where statistically significant heterogeneity was found $(p<0.05)$, the random effects model was used to combine results. ${ }^{11} 12$ Publication bias was assessed using the Egger test, and where statistically significant bias was found, the trim and fill method was used to adjust for it. ${ }^{13} 14$ All analyses were performed using Stata V.10 (StataCorp, College Station, Texas, USA).

\section{RESULTS}

\section{Search results}

The literature search yielded 2243 non-duplicated articles, of which 2063 were excluded on the basis of their title or abstract. Reviewing the full manuscript of the remaining 180 articles led to further exclusions according to the criteria detailed above (see figure 1 for flowchart). After hand searches and subsequent application of NOS quality criteria (see online supplementary tables S1S3), the final number of articles included in the analysis was 23 .

\section{Osteoporosis and osteopenia in PD}

Two studies were included in the analysis that reported the diagnosis of osteoporosis in patients with PD versus healthy controls (see figure 2). The combined OR for osteoporosis in PD patients was $2.61 ; 95 \%$ CI 1.69 to 4.03 (see online supplementary table S4A).

A further four studies reported gender comparisons of osteoporosis/osteopenia in patients with PD. The combined OR for osteoporosis in male PD patients compared with female patients was $0.32 ; 95 \%$ CI 0.18 to 0.58 and for osteopenia was 0.64 ; 95\% CI 0.35 to 1.16 (see figure 2). When data for osteoporosis and osteopenia were combined, the OR for male PD patients versus female PD patients was 0.45 ; $95 \%$ CI 0.29 to 0.68 (see online supplementary table $\mathrm{S} 4 \mathrm{~B}, \mathrm{C})$.

\section{BMD in PD}

Fourteen included studies reported on BMD in PD patients (total number of patients $n=938$ ) and controls $(n=15050)$ (see figure 3 and online supplementary table S5). PD patients had significantly lower BMD than controls; overall combined mean difference, -0.06 ; $95 \%$ CI -0.08 to -0.03 . The overall figure comprised significant differences in the BMD of patients and controls at the femoral neck, lumbar spine, total hip and total body, as well as non-significant differences at the trochanter and Ward's triangle. The combined mean difference was $-0.08,95 \%$ CI -0.13 to -0.02 for the femoral neck; $-0.09,95 \%$ CI -0.15 to -0.03 for the lumbar spine; and $-0.05,95 \%$ CI -0.07 to -0.03 for total hip. Subgroup gender analysis showed female PD patients had

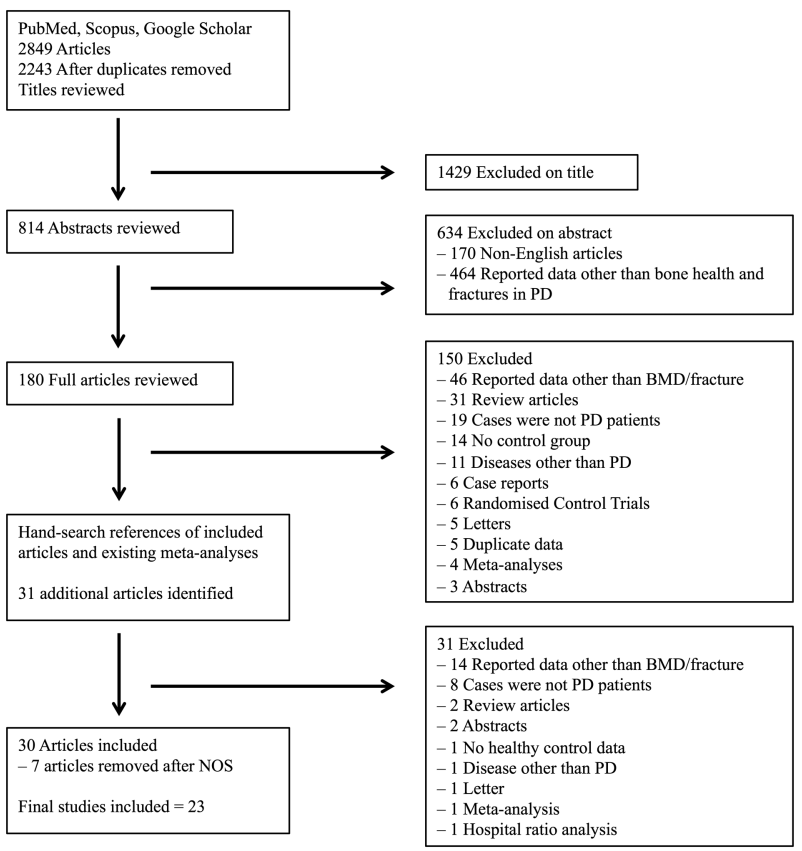

Figure 1 Flowchart of studies included and excluded. PD, Parkinson's disease; BMD, bone mineral density. 


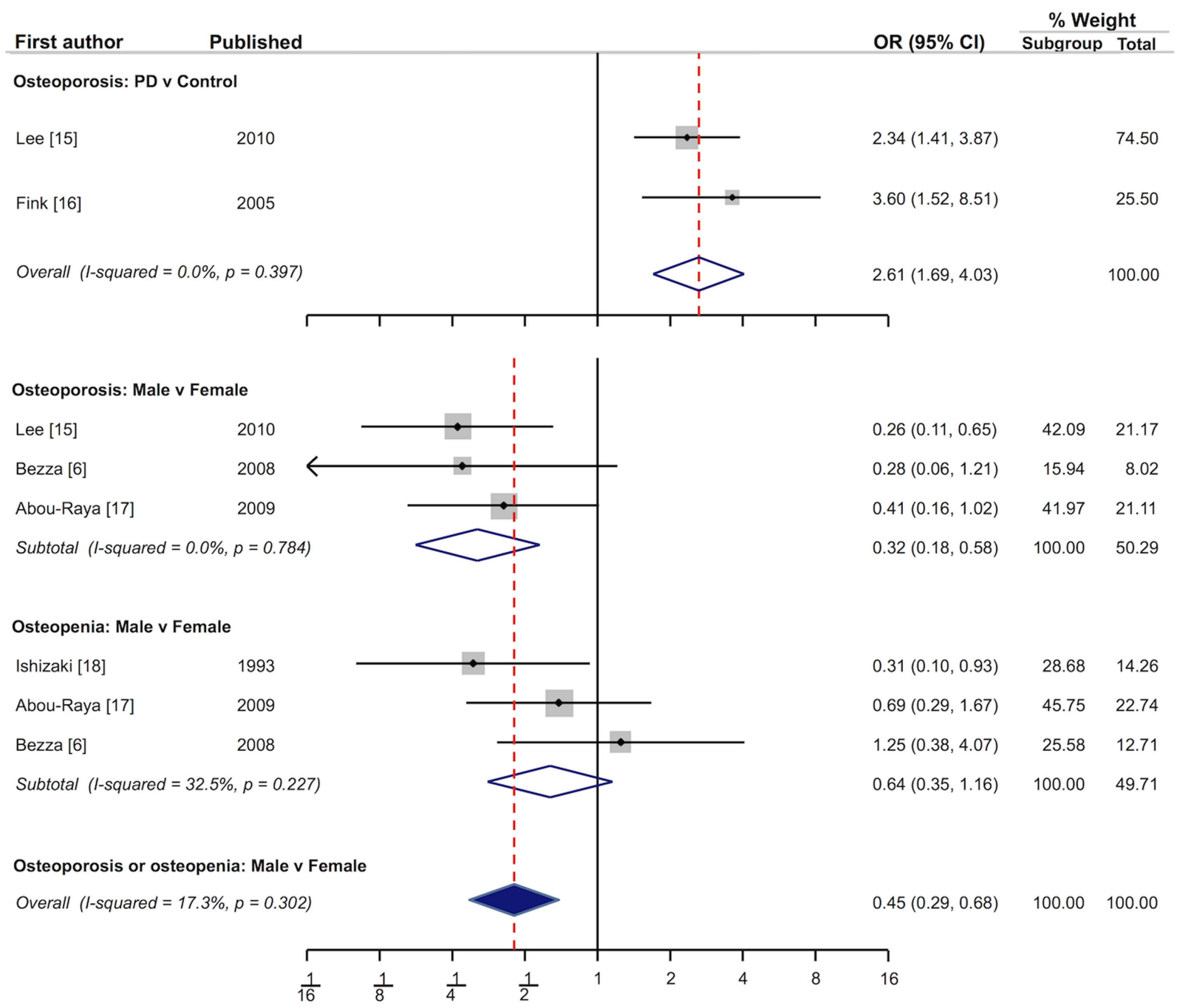

Figure 2 Pooled estimate of $\mathrm{OR}$ and $95 \% \mathrm{Cl}$ of Parkinson's disease (PD) and osteoporosis and osteopenia in male versus female patients.

lower BMD at all body sites compared with male PD patients (data not shown).

Five of these 14 studies and one additional study reported Z-scores (see figure 4 and online supplementary table S6). Patients had significantly lower Z-scores than controls; overall combined mean difference, $-0.75 ; 95 \% \mathrm{CI}-1.00$ to -0.51 . This comprised mean difference of $-1.03 ; 95 \%$ CI -1.31 to -0.74 for femoral neck; $-0.57,95 \%$ CI -0.67 to -0.48 for lumbar spine; and $-0.59 ; 95 \% \mathrm{CI}-0.82$ to -0.35 for total body.

Three of the 14 studies reported T-scores (see figure 5 and online supplementary table S7). PD patients had significantly lower T-scores than controls; overall combined mean difference, $-1.05 ; 95 \%$ CI -1.26 to -0.84 . Separately, the T-scores for lumbar spine, femoral neck and total hip were all significantly lower in PD patients than controls.

\section{Fracture risk in PD}

Nine of the included studies reported data pertaining to fracture risk in PD (see figure 6 and online supplementary table S8). The combined effect size was 2.28 ; $95 \%$ CI 1.83 to 2.83 . Of these, three studies had a combined HR of 2.10 , 95\% CI 1.55 to 2.86; four studies had a combined OR of $4.01,95 \%$ CI 1.77 to 9.04; and two studies had a combined RR of 2.13 , 95\% CI 1.68 to 2.69 . Significant publication bias was noted in studies reporting fracture risk $(\mathrm{p}=0.042)$. The trim and fill method was used to correct for this yielding a new OR of $1.93 ; 95 \% \mathrm{CI} \%$ 1.56 to 2.40 .

\section{DISCUSSION}

The best-established and generalisable risk factors for osteoporosis include age, gender, steroid therapy, low BMI, sedentary lifestyle and smoking. ${ }^{2}$ More recently, neurological diseases have emerged as important causes of secondary osteoporosis. The GLOW found PD to have the strongest association with fractures above all other studied characteristics. ${ }^{4}$

The purpose of this systematic review and meta-analysis was to fully explore the published literature on the risk of osteoporosis, reduction in BMD and fracture risk in patients with PD. The results show that PD patients have significantly increased risk of osteoporosis and osteopenia, and that female patients are more severely affected than male patients. This gender difference in osteoporosis is consistent with that observed in the wider non-PD population. Certain female factors may increase risk of osteoporosis with endocrine and nutritional factors playing an important role.

Invernizzi et $a l^{32}$ reported that osteoporosis and osteopenia affect $91 \%$ of female and $61 \%$ of male patients. Additionally, Schneider et $a l^{23}$ showed female PD patients to have a $7.3 \%$ 
Through combining data from studies using objective measurements of BMD using DEXA imaging only, PD patients were found to have reduced BMD across a wide range of body regions when compared with healthy controls. In addition, combining data from studies that reported T-scores and Z-scores specifically gave a similar conclusion that BMD was reduced in PD patients compared with controls. Again, gender differences in the PD patient population were apparent. The reduction in BMD in PD patients has been previously reported as being more apparent at the lumbar spine and femoral neck and greatest in older women with advanced disease. ${ }^{7}$ Our findings were in agreement with this observation, with PD patients having most marked changes in BMD levels in the lumbar spine and femoral neck regions. intuitive given the well-established gender differences in those without PD.
(95\% Cl)

Weight

First author

Published

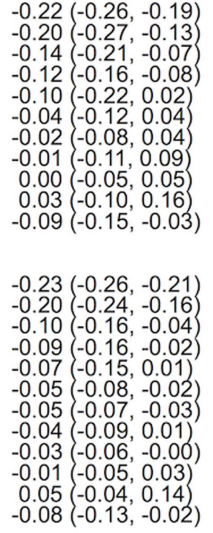

$\begin{array}{rr}11.40 & 2.44 \\ 10.29 & 2.09 \\ 10.48 & 2.15 \\ 11.35 & 2.43 \\ 8.25 & 1.53 \\ 9.69 & 1.92 \\ 10.76 & 2.24 \\ 9.00 & 1.72 \\ 10.95 & 2.29 \\ 7.83 & 1.43 \\ 100.00 & 20.23\end{array}$

$\begin{array}{ll}9.71 & 2.58 \\ 9.33 & 2.40 \\ 8.91 & 2.22\end{array}$

$\begin{array}{ll}8.44 & 2.03 \\ 8.21 & 1.94\end{array}$

$\begin{array}{ll}9.54 & 2.57 \\ 9.71 & 2.49\end{array}$

$9.06 \quad 2.28$

$9.42 \quad 2.44$

$\begin{array}{rr}8.03 & 1.87 \\ 100.00 & 25.36\end{array}$

$-0.09(-0.15,-0.03)$

(1)

$-0.06(-0.09,-0.03$

$-0.04(-0.08,0.00)$

$0.01(-0.04,0.06)$
$-0.05(-0.07,-0.03)$

$-0.06(-0.12,0.00)$

$-0.04(-0.09,0.01$

$-0.04(-0.08,-0.01)$

32.61
46.04

21.35
100.00

2.16
2.28
1.97

6.41

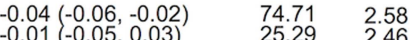

$-0.03(-0.05,-0.01) \quad 100.00 \quad 5.04$

$\begin{array}{lll}-0.08(-0.15,-0.01) & 16.93 & 2.12 \\ -0.07(-0.12,-0.02) & 20.06 & 2.28 \\ -0.03(-0.06,0.00) & 25.03 & 2.48 \\ -0.01(-0.05,0.03) & 24.11 & 2.45 \\ 0.09(0.01,0.17) & 13.87 & 1.93\end{array}$

$-0.08(-0.16,0.00) \quad 51.52 \quad 1.91$

$\begin{array}{rrr}0.04(-0.05,0.13) & 48.48 & 1.87 \\ -0.02(-0.08,0.04) & 100.00 & 3.78\end{array}$

$\begin{array}{lll}-0.03(-0.05,-0.01) & 50.14 & 2.57\end{array}$

$\begin{array}{rrr}-0.02(-0.04,-0.00) & 100.00 \quad 5.13\end{array}$

$\begin{array}{lll}-0.02(-0.07,0.02) & 100.00 \quad 2.40\end{array}$

$-0.04(-0.09,0.01) \quad 49.48 \quad 2.29$

$\begin{array}{lll}-0.01(-0.05,0.02) & 100.00 \quad 4.58\end{array}$

$-0.06(-0.08,-0.03)$ 


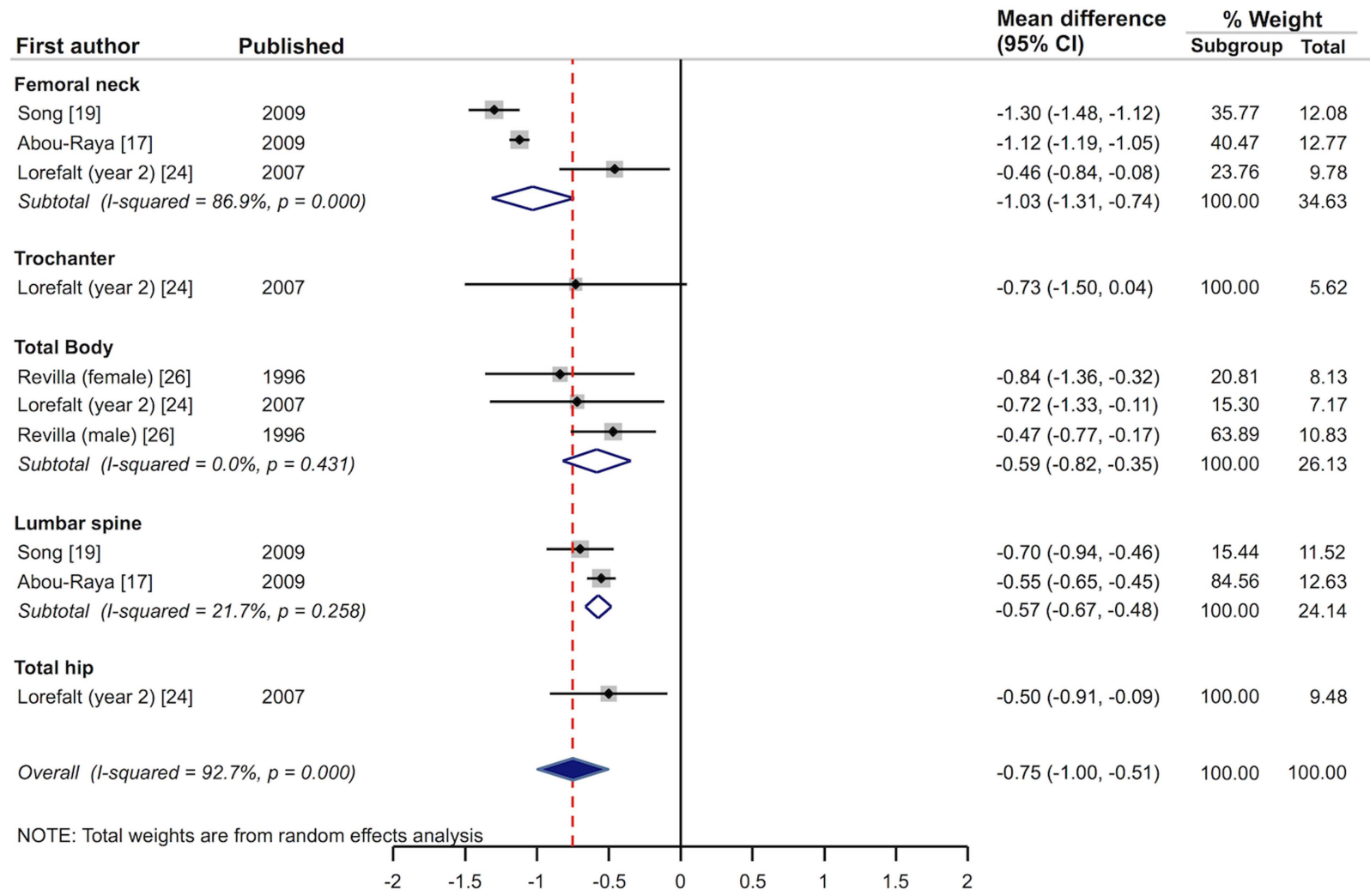

Figure 4 Mean difference and 95\% Cl of Parkinson's disease and bone mineral density Z-score.

Deterioration in bone health in patients with PD can have grave consequences and a significant increase in fracture risk in this patient group compared with healthy controls was observed. Factors that contribute to loss of BMD in PD include vitamin D deficiency with secondary hyperparathyroidism, reduced sunlight exposure, disease duration and severity, age and low body mass index. Factors that increase risk of fractures from falls in the context of reduced BMD include postural instability, orthostatic hypotension, motor fluctuations, cognitive impairment and physical deconditioning. In all, 50\% of PD patients report falling more than once during a 3-month period and $13 \%$ report falling more than once a week. ${ }^{36} 37$

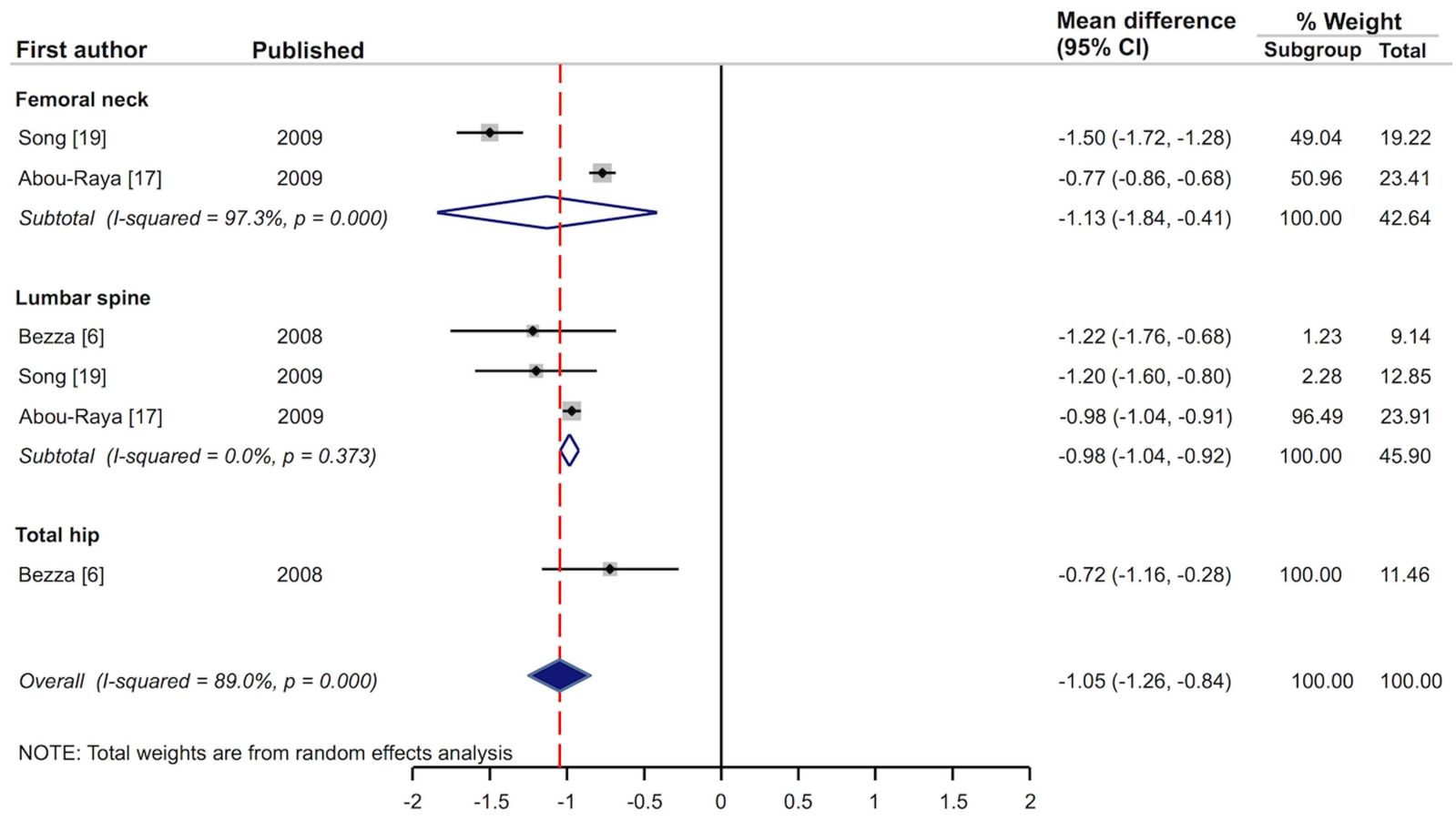

Figure 5 Mean difference and $95 \% \mathrm{Cl}$ of Parkinson's disease and bone mineral density T-score. 
The most common fracture is that of the hip, which accounts for $50 \%$ of all fractures in PD patients. ${ }^{38}$ In non-PD subjects, the most common fragility fractures are vertebral. ${ }^{2}$ The prevalence of vertebral fractures may be underestimated in PD as the typical or expected stooped posture may not solicit investigation for osteoporosis (figure 7). Hip fractures are associated with higher medical costs and greater morbidity than all other osteoporotic fractures. ${ }^{39}$ They also carry a 1-year mortality rate of $30 \%$. ${ }^{40}$ The predisposition for hip fractures in PD may reflect the nature of the falls themselves in that they are often in a sideways or backward direction. ${ }^{29} 31$ Elderly subjects with a rapid gait are more likely to fall forward while those with a shuffling gait tend to fall backward or to the side and suffer a hip fracture. $^{4142}$ Lower BMD levels at the hip may also contribute to the excess rate of hip fractures in PD patients. Of interest is a previous community-based study of PD patients showing that falls risk was significantly greater in patients with dyskinesia and tended to be greater in those with motor fluctuations. ${ }^{43}$ The nine studies that were included in the fracture meta-analysis were reviewed further to see whether an explanation for the difference in fracture site could be determined. None of the studies provided information on motor fluctuations, two provided information on falls and one provided information on walking speed showing that patients were slower than controls and more likely to fall. The reason for the excess risk of hip fracture in PD is an important matter for further study.

Levodopa is central to the drug treatment of PD, but has been implicated as an independent risk factor for fracture and reduced BMD in some studies and the risk may be dosedependent. $^{41}{ }^{44}$ Alongside a possible deleterious effect on
BMD, levodopa improves some motor deficits in PD but tends not to improve postural stability, meaning that patients are potentially more mobile but are perhaps at an increased risk of falls. Side effects of levodopa such as orthostatic hypotension, visual hallucinations and excessive daytime somnolence can further increase risk of falls. ${ }^{41}$ Levodopa can also induce hyperhomocysteinemia which has been reported as a potential risk factor for fractures. ${ }^{45}$ Furthermore, a significant proportion of patients with PD suffer from depression and it is noteworthy that the concomitant use of antidepressants with levodopa has been associated with a threefold to fivefold increase in the risk of hip/femur fractures. ${ }^{41} 46$ Antidepressants inhibit serotonin transport systems and have a detrimental effect on the microarchitecture of the bone and thus reduce BMD. ${ }^{47}$

The management of fracture risk is an important aspect of the holistic care of patients with PD. The National Institute of Health and Care Excellence recommends that assessment with either FRAX $^{48}$ or Qfracture ${ }^{27}$ risk calculators should be 'considered' in all patients with possible secondary osteoporosis and should be used to determine those who should undergo formal BMD measurement using dual-energy X-ray absorptiometry (DEXA) imaging. ${ }^{3}$ The treatment of osteoporosis should follow local guidelines and should be considered on an individual basis. Bisphosphonates are important in the treatment of osteoporosis but side effects such as nausea are relatively common. Correcting vitamin D and calcium levels using supplementation should also be routine in these patients.

Non-pharmacological measures such as exercise programmes, dietary advice, smoking cessation, regular medication review, occupational therapy and physiotherapy assessment, visual 


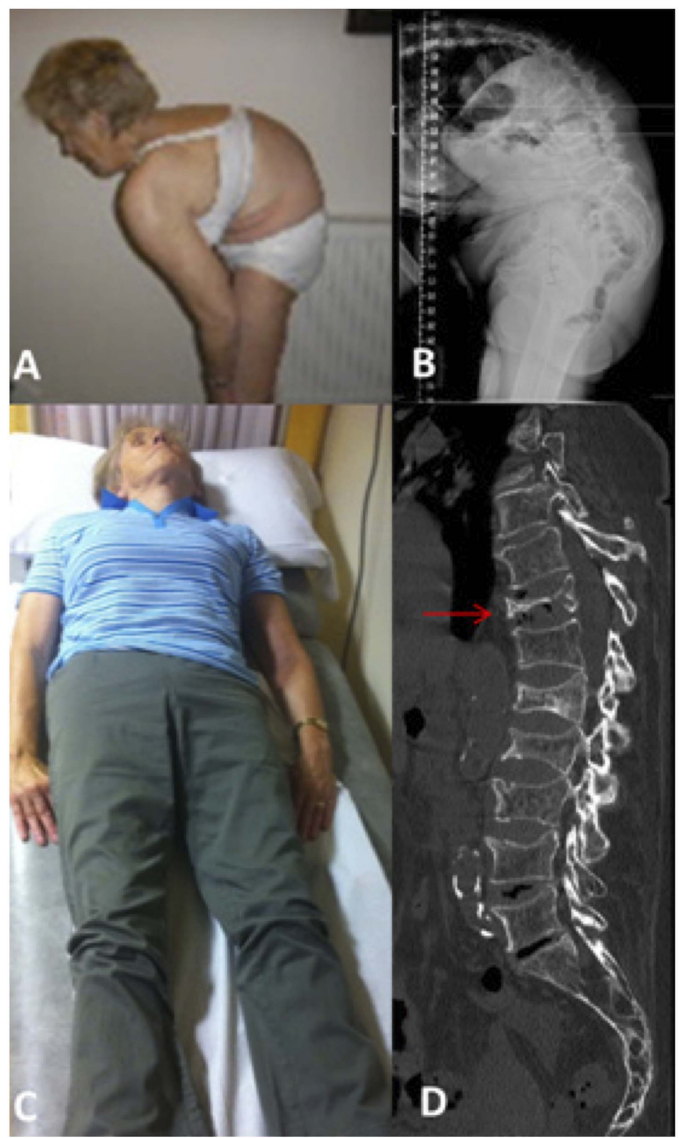

Figure 7 Osteoporotic collapse in a Parkinson's disease patient with camptocormia. This patient has a completely collapsed posture on standing ( $\mathrm{A}$ and $\mathrm{B}$-standing lateral radiograph). When supine, there is normal sagittal alignment of the spine and no flexed joint contractures (C). Supine CT imaging through the midline demonstrates several osteoporotic vertebral fractures. There is complete wedging of T11 (red arrow) and partial collapse of lumbar vertebrae 1-3 (D).

assessment, and falls education and risk management can contribute to reducing falls risk. Specific exercise programmes focusing on strength, balance and flexibility reduce falls rate and risk in community dwelling elderly people. ${ }^{49}$ Ultimately, an integrated approach including falls risk assessment, fracture risk assessment and investigations into secondary causes of osteoporosis in PD patients is needed to prevent falls and fractures in these patients.

There were a number of identifiable limitations when undertaking this review. We restricted our search to articles written in English and therefore reports written in other languages were not included in the analysis. However, this was an important decision since the quality of the studies included was a key consideration and could not be assessed adequately if the authors reviewed non-English articles and their abstracts. Even carefully planned search strategies can miss articles of interest and underestimate the available published literature. To overcome this, we used overlapping search terms, three indexing databases and hand searched the reference list of suitable articles. Reassuringly, the hand search yielded no additional suitable articles that could be included in the final analysis, suggesting that our electronic search strategy was adequate. By following this sequence, we have taken numerous steps to ensure that missing information was kept to a minimum. Low quality studies may distort the overall findings of meta-analysis. We therefore devised and followed stringent inclusion and exclusion criteria, followed by the additional step of employing a recognised quality scoring system for the remaining articles, the NOS scale. ORs, HRs and RRs were considered to be equivalent for the purposes of combining and analysing data. They are not equivalent, however, and in addition some articles did not report an OR and simply gave the raw data. In this situation, two authors independently calculated the OR from the raw data and then cross-referenced their answers. Significant heterogeneity was observed in most of the analyses. This is expected because of differences between individual studies in, for example, study population characteristics and whether crude or adjusted risk estimates were reported.

In conclusion, we have used a comprehensive systematic review and meta-analysis to demonstrate a significant positive association in risk of osteoporosis, reduction in BMD and risk of fractures in patients with PD. Further research is required to look at the basic mechanisms that underpin these observations, as well as more detailed study of the epidemiology, leading onto strategies for pharmacological and non-pharmacological primary and secondary prevention.

Contributors KMT, AJN and KMD: design and conceptualisation of the study, acquisition of data, analysis of the data, interpretation of the data, drafting the manuscript, approval of final version. JB: analysis of the data, interpretation of the data, drafting the manuscript, approval of final version. RD: design and conceptualisation of the study, approval of final version. AJL: design and conceptualisation of the study, interpretation of the data, revision of the manuscript for intellectual content, approval of final version.

Competing interests KMT reports no disclosures. AJN: Travel support from the National Institute of Health Research for presentations at MDS Congresses and Association of British Neurologists Meetings; grants from Parkinson's UK Innovation Grant (K-1006), Parkinson's UK Career Development Award (F-1201), National Institute of Health Research Academic Clinical Fellowship, Élan/Prothena Pharmaceuticals and GE Healthcare. KMD: beneficiary of a Reta Lila Weston Scholarship and received a Parkinson's UK Innovation Grant (K-1010). JPB reports no disclosures. RD reports no disclosures. AJL: Board membership for Novartis, Teva, Meda, Boehringer Ingelheim, GSK, Ipsen, Lundbeck, Allergan, Orion, BIAL, Noscira, Roche; consultancy for Genus; grants from PSP Association, Weston Trust-Reta Lila Howard Foundation; speaking fees from Novartis, Teva, Meda, Boehringer Ingelheim, GSK, Ipsen, Lundbeck, Allergan, Orion, BIAL, Noscira and Roche.

\section{Patient consent Obtained.}

Provenance and peer review Not commissioned; externally peer reviewed.

Open Access This is an Open Access article distributed in accordance with the Creative Commons Attribution Non Commercial (CC BY-NC 3.0) license, which permits others to distribute, remix, adapt, build upon this work non-commercially, and license their derivative works on different terms, provided the original work is properly cited and the use is non-commercial. See: http://creativecommons.org/ licenses/by-nc/3.0/

\section{REFERENCES}

1 de Lau LML, Giesbergen PCLM, de Rijk MC, et al. Incidence of parkinsonism and Parkinson disease in a general population: the Rotterdam Study. Neurology 2004:63:1240-4.

2 Osteoporosis: primary prevention. NICE guidance. http://www.nice.org.uk/guidance/ TA160 (accessed 24 Aug 3013)

3 Osteoporosis: assessing the risk of fragility fracture. NICE guidance. http:// publications.nice.org.uk/osteoporosis-assessing-the-risk-of-fragility-fracture-cg146 (accessed 24 Aug 2013).

4 Dennison EM, Compston JE, Flahive J, et al. Effect of co-morbidities on fracture risk: findings from the Global Longitudinal Study of Osteoporosis in Women (GLOW). Bone 2012;50:1288-93.

5 Dobson R, Yarnall A, Noyce AJ, et al. Bone health in chronic neurological diseases: a focus on multiple sclerosis and parkinsonian syndromes. Pract Neurol 2013;13:70-9.

6 Bezza A, Bezza Z, Ouzzif $H$, et al. Prevalence and risk factors of osteoporosis in patients with Parkinson's disease. Rheumatol Int 2008;28:1205-9.

7 Vaserman N. Parkinson's disease and osteoporosis. Joint Bone Spine 2005; 72:282-8 
8 Moher D, Liberati A, Tetzlaff J, et al. Preferred reporting items for systematic reviews and meta-analyses: the PRISMA statement. BMJ 2009;339:b2535.

9 Gibb WR, Lees AJ. The relevance of the Lewy body to the pathogenesis of idiopathic Parkinson's disease. J Neurol Neurosurg Psychiatry 1988;51:745-52.

10 Ottawa Hospital Research Institute. NOS criteria. http://www.ohri.ca/programs/ clinical_epidemiology/oxford.asp (accessed 28 Aug 2013).

11 Higgins JPT, Thompson SG, Deeks JJ, et al. Measuring inconsistency in meta-analyses. BMJ 2003;327:557-60.

12 DerSimonian R, Laird N. Meta-analysis in clinical trials. Control Clin Trials 1986:7:177-88.

13 Egger M, Smith GD, Phillips AN. Meta-analysis: principles and procedures. BMJ 1997:315:1533-7.

14 Duval S, Tweedie R. Trim and fill: a simple funnel-plot-based method of testing and adjusting for publication bias in meta-analysis. Biometrics 2000:56:455-63.

15 Lee SH, Kim MJ, Kim BJ, et al. Hyperhomocysteinemia due to levodopa treatment as a risk factor for osteoporosis in patients with Parkinson's disease. Calcif Tissue Int 2010;86:132-41.

16 Fink HA, Kuskowski MA, Taylor BC, et al. Association of Parkinson's disease with accelerated bone loss, fractures and mortality in older men: the Osteoporotic Fractures in Men (MrOS) study. Osteoporos Int 2008;19:1277-82.

17 Abou-Raya S, Helmii M, Abou-Raya A. Bone and mineral metabolism in older adults with Parkinson's disease. Age Ageing 2009;38:675-80

18 Ishizaki F, Harada T, Katayama S. Relationship between osteopenia and clinical characteristics of Parkinson's disease. Mov Disord 1993;8:507-11.

19 Song IU, Kim JS, Lee SB, et al. The relationship between low bone mineral density and Parkinson's disease in a Korean population. J Clin Neurosci 2009;16: 807-9.

20 Kamanli A, Ardicoglu 0, Ozgocmen A, et al. Bone mineral density in patients with Parkinsons disease. Aging Clin Exp Res 2007;20:277-9.

21 Taggart $\mathrm{H}$, Crawford V. Reduced bone density of the hip in elderly patients with Parkinson's disease. Age Ageing 1995;24:326-8

22 Lam K, Li M, Mok L. A case control study on bone mineral density in Chinese patients with Parkinson's disease. Parkinsonism Relat Disord 2010;16:471-4.

23 Schneider JL, Fink HA, Ewing SK. The association of Parkinson's disease with bone mineral density and fracture in older women. Osteoporos Int 2008;19:1093-7.

24 Lorefalt B, Toss G, Granerus A-K. Bone mass in elderly patients with Parkinsons disease. Acta Neuro/ Scand 2007;116:248-54.

25 Fernandez MC, Parisi MS, Diaz SP. A pilot study on the impact of body composition on bone and mineral metabolism in Parkinson's disease. Parkinsonism Relat Disord 2007;13:355-8.

26 Revilla M, de la Sierra G, Aguado F, et al. Bone mass in Parkinson's disease: a study with three methods. Calcif Tissue Int 1996;58:311-15

27 Hippisley-Cox J. Derivation and validation of updated QFracture algorithm to predic risk of osteoporotic fracture in primary care in the United Kingdom: prospective open cohort study. BMJ 2012;344:e3427.

28 Chen Y, Cheng PY, Wu SL, et al. Parkinson's disease and risk of hip fracture: an 8-year follow-up study in Taiwan. Parkinsonism Relat Disord 2012;18:506-9.
29 Genever RW, Downes TW, Medcalf P. Fracture rates in Parkinson's disease compared with age- and gender-matched controls: a retrospective cohort study. Age Ageing 2005:34:21-4.

30 Leibson CL, Maraganore DM, Bower JH. Comorbid conditions associated with Parkinson's disease: a population-based study. Mov Disord 2006;21:446-55.

31 Johnell O, Melton LJ III, Atkinson EJ, et al. Fracture risk in patients with parkinsonism: a population-based study in Olmsted County, Minnesota. Age Ageing 1992;21:32-8.

32 Invernizzi M, Carda S, Viscontini GS, et al. Osteoporosis in Parkinson's disease. Parkinsonism Relat Disord 2009:15:339-46.

33 Zhao Y, Shen L, Ji HF. Osteoporosis risk and bone mineral density levels in patients with Parkinson's disease: a meta-analysis. Bone 2013;52:498-505.

34 Hagenau T, Vest R, Gissel TN. Global vitamin D levels in relation to age, gender, skin pigmentation and latitude: an ecologic meta-regression analysis. Osteoporosis Int 2009;20:133-40.

35 Wooten GF, Currie LJ, Bovbjerg VE, et al. Are men at greater risk for Parkinson's disease than women? J Neurol Neurosurg Psychiatry 2004;75:637-9.

36 Pickering RM, Grimbergen YAM, Rigney U, et al. A meta-analysis of six prospective studies of falling in Parkinson's disease. Mov Disord 2007:22:1892-900.

37 Koller WC, Glatt S, Vetere-Overfield B, et al. Falls and parkinson's disease. Clin Neuropharmacol 1989:2:98-105

38 Williams DR, Watt HC, Lees AJ. Predictors of falls and fractures in bradykinetic rigid syndromes: a retrospective study. J Neurol Neurosurg Psychiatry 2006;77:468-73.

39 El Maghraoui A, Koumba BA, Jroundi I, et al. Epidemiology of hip fractures in 2002 in Rabat, Morocco. Osteoporos Int 2005; 16:597-602.

40 Wiles MD, Moran CG, Sahota 0, et al. Nottingham Hip Fracture Score as a predictor of one year mortality in patients undergoing surgical repair of fractured neck of femur. Br J Anaesth 2011;106:501-4.

41 Arbouw MEL, Movig KLL, van Staa TP, et al. Dopaminergic drugs and the risk of hip or femur fracture: a population-based case-control study. Osteoporos Int 2011;22:2197-204.

42 Graafmans W, Ooms M, Bezemer $\mathrm{P}$, et al. Different risk profiles for hip fractures and distal forearm fractures: a prospective study. Osteoporos Int 1996;6:427-31.

43 Schrag A, Quinn N. Dyskinesias and motor fluctuations in Parkinson's disease a community-based study. Brain 2000;123:2297-305.

44 Vestergaard P, Rejnmark L, Mosekilde L. Fracture risk associated with Parkinsonism and anti-Parkinson drugs. Calcif Tissue Int 2007;81:153-61.

45 Sato $Y$, Iwamoto J, Kanoko T, et al. Homocysteine as a predictive factor for hip fracture in elderly women with Parkinson's disease. Am J Med 2005;118:1250-55.

46 Lieberman A. Depression in Parkinson's disease-a review. Acta Neurol Scand 2006;113:1-8.

47 van den Brand MW, Samson MM, Pouwels S, et al. Use of anti-depressants and the risk of fracture of the hip or femur. Osteoporos Int 2009;20:1705-13.

48 Kanis JA, Johnell 0, Oden A, et al. FRAX and the assessment of fracture probability in men and women from the UK. Osteoporos Int 2008;19:385-97.

49 Gillespie LD, Robertson MC, Gillespie WJ, et al. Interventions for preventing falls in older people living in the community. Cochrane Database Syst Rev 2009:(2):CD007146. 